\title{
Simulation, design and structure of ITS models for supporting evacuation in smart cities
}

\author{
M. L. De Maio, G. Musolino, A. Polimeni \& A. Vitetta \\ Dipartimento di Ingegneria dell'Informazione, delle Infrastrutture e \\ dell'Energia Sostenibile, DIIES, Università degli Studi Mediterranea di \\ Reggio Calabria, Italy
}

\begin{abstract}
Many technologies have been developed to assist mobility in a smart city; however, there is a lack of models to support the technology. In this paper, a framework is proposed to integrate a model system into an Intelligent Transport System (ITS) environment. The framework allows us to integrate the information provided by the technology in order to configure the transport network. Models considered are both simulation models (i.e. assignment) and design models (vehicle routing). This framework could be applied to support managing evacuation operations in a city.

Keywords: smart city, Intelligent Transportation Systems, path choice, vehicle routing, design models.
\end{abstract}

\section{Introduction}

A smart city is a city well performing in six characteristics, built on the 'smart' combination of endowments and activities of self-decisive, independent and aware citizens (http://www.smart-cities.eu/model.html). Smart mobility is a relevant element in smart city definition. Although many studies aimed to support mobility are mainly technology-oriented, the modelling system is not enough developed. Technologies able to support the mobility in a smart city are called Intelligent Transportation Systems (ITS): their applicability range from the vehicles survey to information for users.

ITS can be used to improve the mobility in a city; but ITS introduction in a transport system needs a preliminary system analysis to appraisal their effects [1]. The analysis in this paper regards the models applicable into an ITS 
environment in order to simulate and design an urban traffic network. The aim is to support city managers, municipal authorities [2] and users during an evacuation, it is necessary to guide users toward a safe area. If users move with private motorized modes, information in real time on transport network is crucial to speed up the evacuation. For example, users can get information about the path to follow or about the nearest safe area. Similar considerations can be made considering the case in which users are evacuated using transit modes (i.e. bus) or, if it is necessary, an ambulance. In this case, drivers should receive from ITS system information about paths and user's location. A compact scheme to represent the aim of the simulation of users of a transportation system is reported in Figure 1 that will be detailed in the next sections of the paper. Starting from the current transport system (network topology and costs, signal setting), ITS depicts the current traffic conditions at time t. The behavioural simulation includes all the procedures allowing forecasting the system status after a time slice $\Delta \mathrm{t}$. If the transport system conditions are surveyed at instant $\mathrm{t}$ and the behavioural simulation require a computing interval $\Delta \mathrm{t}_{0}$, the approach done the forecasted traffic conditions until the time $\mathrm{t}+\Delta \mathrm{t}$.

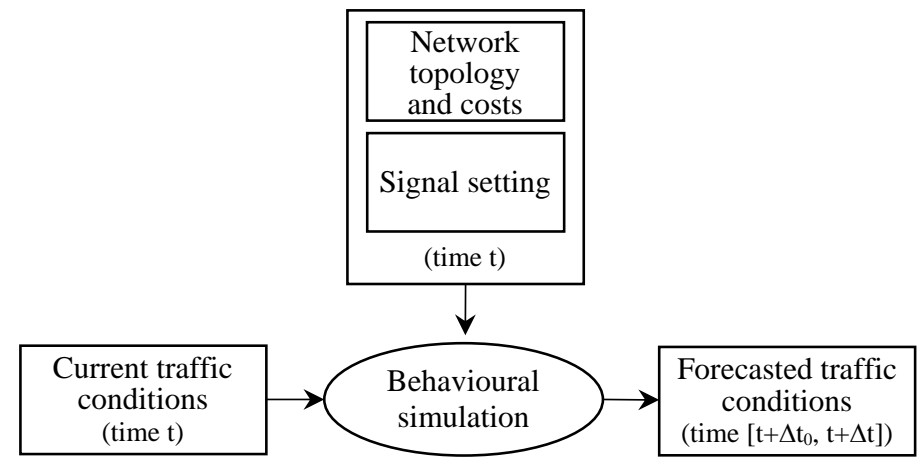

Figure 1: Compact system simulation.

The paper is structured as follows. Section 2 reports on a literature review on smart mobility and transport system simulation models. In Section 3 the general framework is discussed. In Section 4 the ITS to survey the system conditions are analysed. Section 5 reports on behavioural simulation, considering three subsections: demand and supply, dynamic traffic assignment, reverse assignment. In Section 6, a design procedure (that includes behavioural simulation) is proposed to solve the vehicle routing problem.

\section{Literature review}

Technologies supporting a smart mobility can be subdivided into devices to survey the transport system and devices to inform users. In first case, the aim is to collect traffic data (i.e. flow, speed, travel time); in second case the aim is to 
know the network performances and to allow forecasting the transport system over time. To make this possible, simulation models are required in order to anticipate the system evolution. One approach to this problem is the use of the Dynamic Traffic Assignment (DTA) models, which simulate the interactions between the time-varying network and time-varying travel demand (in non steady-state traffic conditions). The aim is to capture transport system evolution when travel demand peaks, temporary capacity variations, queue formation and dispersion occur.

DTA models can be classified into analytical (system optimum or user equilibrium) and simulation-based [3, 4]. The former approximate the problem with a system-wide (system optimum) or individual (user equilibrium) objectives, through mathematical formulations and explicit constraints. Yet they represent user behaviour and traffic dynamics in a simplified way. The latter are able to reproduce via simulation the complex inter-temporal demand-supply interactions between time-varying travel demand and the transport network. However, their outputs depend on a large amount of inputs and parameters that need to be estimated. Simulation-based models are generally grouped according to their level of aggregation of user behaviour into macroscopic, mesoscopic and microscopic.

A large literature describes experiences connected to urban traffic monitoring. The traffic flow measures by means of monitoring techniques belong to two classes [5, 6]:

- $\quad$ measures at fixed points of the network with tools like loop detectors and image processing [7];

- measures with floating cars [8] in the network (individual cars, taxis, transit system vehicles).

In the information field, Maerivoet et al. [9] propose a system able to capture the user behavioural giving information related to the travel cost variations, while in [10] is analysed the impact of user information in path choice. In [11] a probe vehicle equipped with a personal digital assistant and GPS antenna provided the link travel time.

\section{General framework}

Figure 2 depicts a general framework where the simulation and design models are integrated into an ITS environment. It describes the behavioural simulation which is operated by means of the Dynamic Traffic Assignment (DTA) and the Reverse Assignment (RA) models.

The survey is based on hardware/software devices [5, 6] able to capture the current traffic conditions on the networks.

The survey is carried out using an automatic monitoring system, which consists on a peripheral survey station close to the traffic scene to be monitored, and a transmission system [12].

The monitoring system provides in real time (on-line data) data related to the current traffic conditions that converge into a dataset (dataset population). They are stored as historical data (off-line data). 


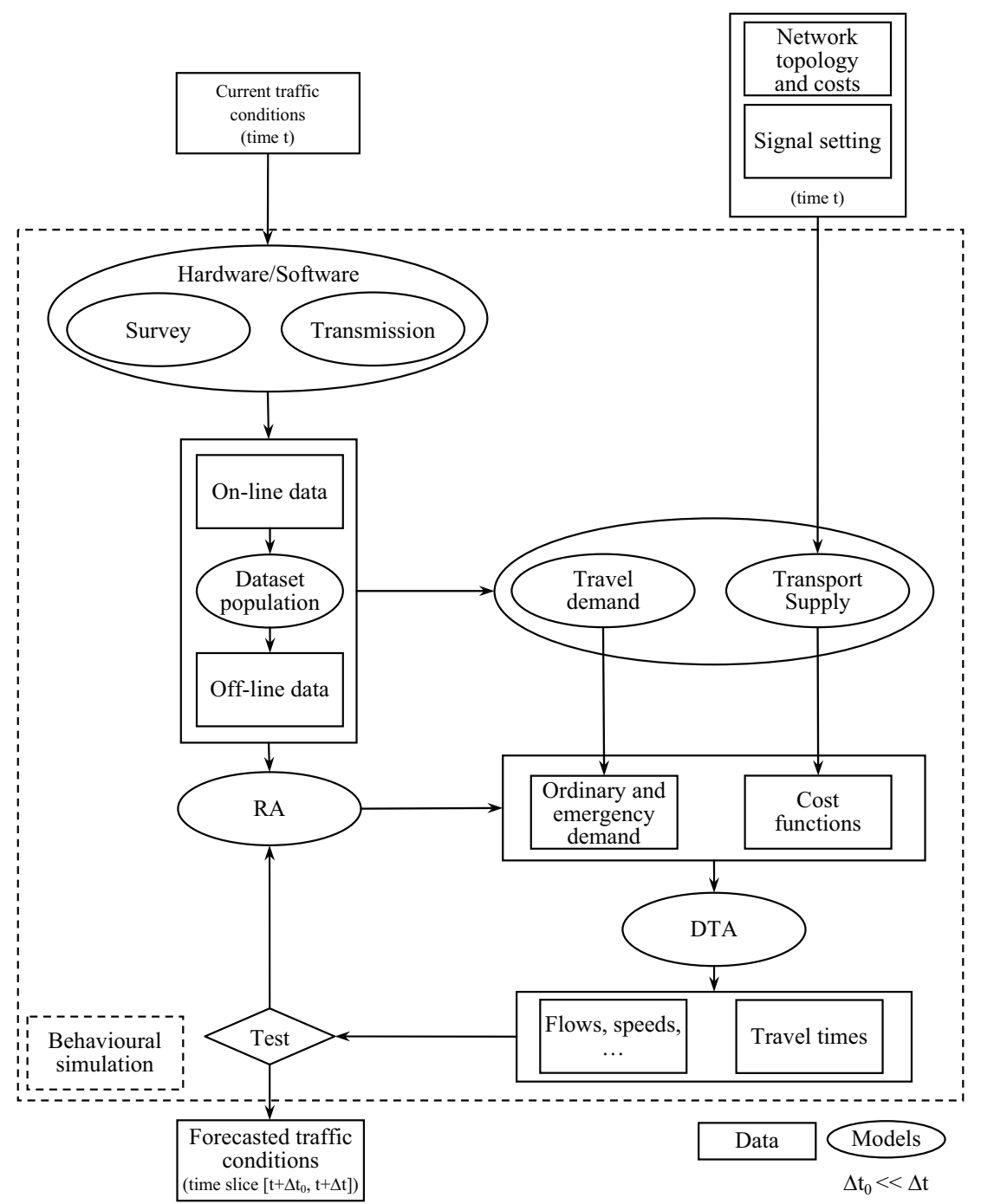

Figure 2: $\quad$ System simulation and design in an ITS environment.

The collected data (both on-line and off-line) are the input for travel demand and transport supply models.

The travel demand model allows us to estimate the users that travel from origins to destinations, with each available mode. In evacuation conditions, two levels of demand must be considered [13, 14]: the ordinary demand (or historical demand), composed by users of the road network that perform systematic trips by motorized modes in the reference time period; the emergency demand, composed by users of the road network that perform trips by motorized modes in 
order to leave from the place where they are in the reference time period and where it is supposed that the catastrophic event will deploy its effects.

The supply model allows obtaining the cost functions. The time spent in the network by a user depends on the network configuration (in terms of lanes allocation, link direction and junction optimization): fix the network configuration; it is possible to define the link cost functions. Generally, the used cost functions, to forecast the link cost, depend both on flow and time $[15,16]$. In an ITS system it is possible to update the function, re-calibrating the parameters considering the real time collected data.

The DTA model has input the demand and the supply. DTA simulates the interaction between travel demand flows and the road transport network in order to estimate time-varying travel times and vehicular flows on a network. Among the outputs, are highlighted flows, speed and travel time.

The output of simulation-based DTA are the input for Reverse Assignment (RA). Other input for the RA coming from ITS system in the form of on-line data and off-line data. Note that the on-line data contribute to populate a database that collect all the observed data.

RA is an approach that makes it possible, starting from the cost and the flow on some links, to obtain the demand and the costs of all links.

The RA models [17] have the following input:

- link flows;

- $\quad$ link performance in terms of costs;

and give as output

- $\quad$ the link cost parameters of the cost-flow functions used in the supply model;

- the value (number of trips) and/or the model parameters of the demand model.

Figure 2 shows the loop existing between the DTA and RA: to correct the link costs and calibrate model parameters, the RA use recursively the output given by the DTA until the convergence.

The output of whole procedure is the forecasted traffic conditions in a time interval $\left[t+\Delta t_{0}, t+\Delta t\right]$, where $\Delta t_{0}$ takes into account the computation time to run the DTA model $\left(\Delta t_{0}<<t\right)$.

The procedure works in time according to the concept of rolling horizon, as detailed in [16].

\section{Brief overview on survey and transmission systems}

In this section, a small overview on survey and transmission systems it is not the core of the paper but a brief description is required to frame the topic.

The survey and transmission system works as follows. Traffic data are detected by sensors and, then, they are stored and transmitted to a remote station. Sensors may be distinguished in relation to the installation procedure (intrusive or non-intrusive) or in relation to the extension of the monitored area (tripwire or vehicle tracking). 
Systems able to detect a specific vehicle, called Automatic Vehicle Location systems (AVL), may be based on a GPS technology, GSM modem, Wi-Fi, radio frequencies.

Data acquired through sensors located in the peripheral station are transmitted with off-line or on-line mode. Because of the recent widespread of technologies, several communication systems can be used in order to give information to users like radio, telephone, mobile, internet, SMS, GSM, Wi-Fi and so on. It is worth distinguishing systems providing information to road users and systems providing information supporting logistic fleets.

Intelligent systems used to provide information to users are called Advanced Traveler Information System (ATIS) [18] and may be distinguished in relation to the kind of information provided: pre-trip or en-route.

Application of ITS in the logistic field concerns the Computerised Vehicle Routing and Scheduling (CVRS) systems which optimize vehicles routes and vehicles allocations. Also in this case, some specific systems are available in emergency conditions: for managing emergency vehicles like ambulances and fire trucks, by sending them radio messages (e.g. about path to be followed [19]). GSM technology was used in [20] and a monitor can be used by the ambulance driver to know the path to be followed as indicated by the control center. The importance of this topic has been stressed in the MAGES project (Mature Applications of Galileo for Emergency Scenario), which describes supports for managing emergencies [21].

\section{Behavioural simulation}

Some models compose the behavioural simulation, to consider some relevant aspects of the transport system. The models considered in this paper, and explained in detail in the next sub-section, are:

- $\quad$ demand and supply models;

- dynamic traffic assignment;

- $\quad$ reverse assignment.

\subsection{Demand and supply models}

The ordinary demand is usually estimated using a multistep approach, considering the emission, the departure time, the distribution and the modal split levels. The emergency demand, can be estimated with a sequential approach (see [22-24]), by considering that in the presence of an ITS, some choice dimensions (i.e. departure time, path choice) can be influenced by the information provided to users.

The demand (ordinary and evacuation), in a time interval $\Delta t$ for an origindestination pair rs, can be expressed per category, as in [22]:

where

$$
d_{\mathrm{rs}}(\Delta t)=d_{\mathrm{rs}}^{R}(\Delta t)+d_{\mathrm{rs}}^{W}(\Delta t)+d_{\mathrm{rs}}^{C}(\Delta t)+d_{\mathrm{rs}}^{S}(\Delta t)+d_{\mathrm{rs}}^{D}(\Delta t)
$$


- $\quad$ R, residents; $W$, employees; $C$, occasional customers; $S$, teachers and pupils; $D$, weak users;

- $\quad d^{R}{ }_{\mathrm{rs}}(\Delta t)$, demand of people belong to resident category;

- $d_{\mathrm{rs}}^{W}(\Delta t)$, demand of people belong to employee category;

- $d_{\mathrm{rs}}^{C}(\Delta t)$, demand of people belong to occasional customer category;

- $d^{S}{ }_{\mathrm{rs}}(\Delta t)$, demand of people belong to schools;

- $d^{D}$ rs $(\Delta t)$, demand of people belong to weak users' category.

Each category can be specified with a descriptive or a behavioural model.

Transport supply could change due to effects of emergency conditions. The network configuration could be modified to make easy the evacuation procedures, changing the use of some links or their direction (as in contraflow operations).

Hence, a modified configuration of the network may be currently available. Users cannot have a complete knowledge of the current state of the network. Considering that users' behaviour is influenced by their experience and knowledge of the network, it is clear the important role played by information in this case. Monitoring systems are necessary to survey and detect changes on the network, with must be used to correct the network configuration, in order to obtain results from assignment simulation (which are consistent with the modified network configuration) and to be diffused to users.

\subsection{Dynamic Traffic Assignment}

Existing within-day DTA models may be employed according to approaches: non-equilibrium or equilibrium. A description of the two approaches is reported in $[5,6,16,25]$. In the case of an equilibrium approach, an iterative process is necessary to find the dynamic equilibrium solution, based on three algorithms [25]: i) an algorithm which calculates route travel times (network loading); ii) a shortest path algorithm which calculates $k$ shortest path and updates path choice set; iii) an algorithm which provides assignment of vehicles to paths in order to simulate the dynamic equilibrium. After completing the three steps described above, it is necessary to come back to the first one: route choices made in the first iteration changed travel times on the network. The iterative procedure is repeated until convergence (if any).

A specific dynamic non-equilibrium model is proposed in [25] in order to simulate path flows related to each $r$ s pair, $d_{\mathrm{rs}}(\Delta t)$, composed by flows on all paths $k$ chosen by users between origin $r$ to destination $s$. Considering time composed by several decision time instants, $\tau^{*}$, the travel demand flow on path $k$ in time $\tau^{*}$ will be $h_{k, \tau *}$. For simplicity's sake here is reported briefly the mathematical formulation for simulation of $d_{\mathrm{rs}}(\Delta t)$, which allows obtaining the traffic flow on link a at time instant $t, f_{a}(\mathrm{t})$ :

$$
f_{a}(x)=\sum_{\tau^{*}=[t, t]} \sum_{(r s)} \sum_{k \in I r s: a \in k} q_{\mathrm{a}}{ }^{k}\left(x \mid \tau^{*}\right) h_{k, \tau^{*}} \quad \mathrm{x} \in\left[\mathrm{t}+\Delta \mathrm{t}_{0}, \mathrm{t}+\Delta \mathrm{t}\right]
$$

where 
- $\quad q_{\mathrm{a}}{ }^{\mathrm{k}}\left(t \mid \tau^{*}\right)$ is the probability of being on link a belonging to path $k$ at the time instant $t$ conditional upon the decision (to choose path $k$ ) at time instant $\tau^{*}$;

- $\quad h_{k, \tau^{*}}=p_{k, \tau^{*}} d_{\mathrm{rs}}(\Delta t)$ is the travel demand flow on path $k$ for each decision time instant $\tau^{*}$;

- $\quad p_{k, \tau^{*}}$ is the probability to choice the path $k$ at instant $\tau^{*}$.

\subsection{Reverse assignment}

The RA was proposed in [17] to re-calibrate link cost functions and update demand values from traffic counts and time measurements on some network links. RA models, starting from observed costs and flows, provide link cost parameters of the cost-flow functions used in the supply model, the value (number of trips) and/or the model parameters of the demand model. A mathematical formulation of RA is provided by [17]:

objective function:

$$
z_{1}\left(\mathbf{d}(\Delta \mathrm{t}), \mathbf{d}^{*}(\Delta \mathrm{t})\right)+z_{2}\left(\mathbf{f}(\mathrm{t}), \mathbf{f}^{*}(\mathrm{t})\right)+z_{3}\left(\mathbf{c}(\mathrm{t}), \mathbf{c}^{*}(\mathrm{t})\right)
$$

control variables:

$\mathbf{d}_{\mathrm{t}}, \mathbf{f}_{\mathrm{t}}, \mathbf{c}_{\mathrm{t}}$

constraints:

$$
\begin{aligned}
& \mathbf{d}(\Delta \mathrm{t}) \geq \mathbf{0} \\
& \mathbf{f}(\mathrm{t}) \geq \mathbf{0} \\
& \mathbf{c}(\mathrm{t}) \geq \mathbf{0} \\
& \mathrm{t} \in\left[\mathrm{t}+\Delta \mathrm{t}_{0}, \mathrm{t}+\Delta \mathrm{t}\right] \\
& \text { user behaviour (as equation (2)) }
\end{aligned}
$$

where

- $\quad \mathbf{d}(\Delta \mathrm{t}), \mathbf{f}(\mathrm{t}), \mathbf{c}(\mathrm{t})$ (demand, flow and cost vector) are time dependent;

- $z(\cdot)$ is a distance function between the observed values and the estimated values for demand $\left(z_{1}\right)$, flow $\left(z_{2}\right)$ and $\operatorname{cost}\left(z_{3}\right)$.

\section{Routes design}

The behavioural simulation is a fundamental component of the approach proposed to design the vehicle routes, which also includes the Network Topology and Signal Setting Design Problem (NTSSDP). Figure 3 shows the recursive procedure that depicts the relationship among the three components. In the design approach, four constraint types are considered:

- law constraints (imposed by administration, i.e. restrict the access in an area);

- technological constraints (imposed by the limitations in the available instruments);

- $\quad$ behavioural constraints (hypothesis on user's behavioural);

- technical constraints (imposed by the design problem, i.e. green time, number of lanes).

The recursive procedure, starting from a system status (i.e. the system status at instant $t$ ) simulate the system with the behavioural simulation obtaining the forecasted traffic estimation data used in NTSSOP to design the network 
topology and the signal setting. Supposing that the current system status is surveyed at instant $t$, the behavioural simulation done the transport system status for a time slice $t+\Delta t$, considering a time slice $\Delta t_{1}$ to solve the behavioural simulation.

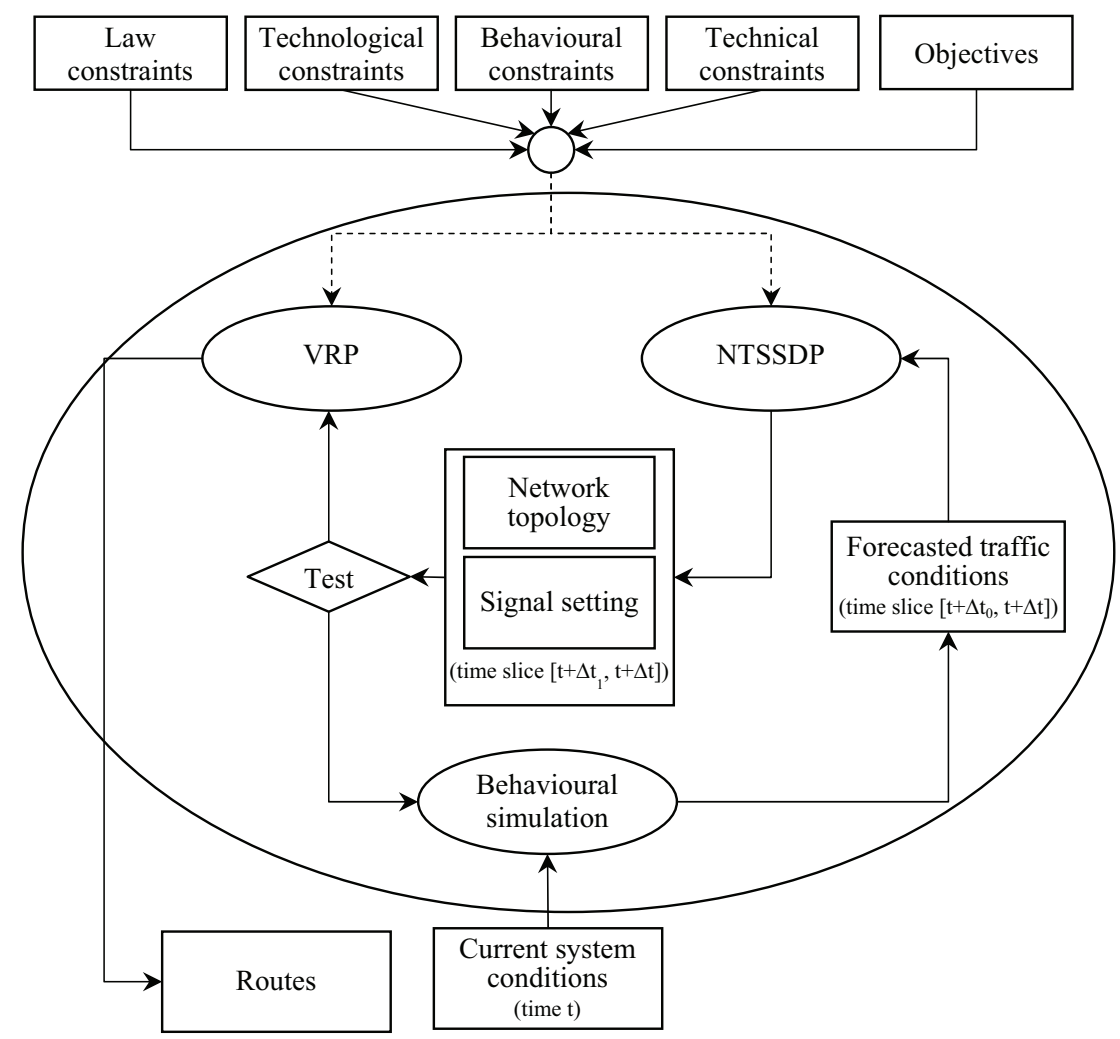

Figure 3: $\quad$ Route design approach

The lane allocation and the link direction define the network topology; due objectives and constraints, the problem can be formulated as an optimum constrained problem. Generally, the design approach is a heuristic, but can be considered hybrid approaches (i.e. an algorithm for topology design another for node optimization). In the network topology design [26] various objectives can be introduced: for example, the evacuation time for all users can be minimized, the route travel time of emergency vehicles can be minimized and so on [26]. A multi-objective problem can be also formulated.

The signal setting consists on defining, for each junction in the network, the cycle and the green time. The problem can be solved simultaneously or after the network topology design, with a global approach or a local approach. 
The procedure is repeated until a test is satisfied (i.e. the value of objective function, the maximum number of iterations).

The output is the network configuration in term of topology and signal setting (that are the first set of output of the whole procedure), that are also the input of VRP. The solution of VRP are the routes for a fleet of vehicle that move in the transportation system.

A joint VRP-NTSSDP formulation is proposed in [26]. The general formulation is as follows:

objective function:

$\mathrm{z}\left(\mathbf{y}^{(\mathrm{TO})}, \mathbf{y}^{(\mathrm{SG})}, \mathbf{\Omega}, \mathbf{f}(\mathrm{t})\right)$

control variables:

$\mathbf{y}^{(\mathrm{TO})}, \mathbf{y}^{(\mathrm{SG})}, \Omega, \mathbf{f}(\mathrm{t})$

constraints:

$$
\begin{aligned}
& \text { technical and structural; } \\
& \text { network connection; } \\
& \text { user behaviour; } \\
& \text { VRP classical constraints }
\end{aligned}
$$

where

- $\mathbf{y}^{(\mathrm{TO})}$ and $\mathbf{y}^{(\mathrm{SG})}$ vectors, the first being related to the network topology (lane allocation), and the second being related to the signal setting (intersection regulation, capacity);

- $\Omega$ matrix, related to the paths used by the vehicles in VRP;

- $\mathbf{f}(\mathrm{t})$, flow vector.

\section{Conclusions}

A general framework where simulation and design models are integrated into on an ITS environment was proposed. The framework is suited to support an evacuation in a smart city. The framework components are:

- the transport system in term of network topology and signal setting, at a generic time;

- $\quad$ the ITS, to capture the actual system conditions.;

- a behavioural simulation, consisting on Dynamic Traffic Assignment (DTA) and Reverse Assignment (RA), to forecast the system status in a time slice.

For each framework component, a formulation was proposed. Finally, a vehicle routing problem with topology and signal setting design was formulated in an integrated model.

\section{Acknowledgements}

Partially supported by National MIUR under PRIN2009 grants $n$. 2009EP3S42_001. 


\section{References}

[1] Cantarella, G.E. Day-to-day dynamic models for Intelligent Transportation Systems design and appraisal. Transport. Res. Part C, 2012.

[2] Russo, F. and Rindone, C., Evaluation methods for evacuation planning. WIT Transactions on the Built Environment, 111, pp. 335-343, 2010.

[3] Peeta S. and Ziliaskopoulos A.K., Foundations of Dynamic Traffic Assignment: the past, the present and the future, Net and Spat Econ, 1(3), pp. 233-265, 2001.

[4] Mahamassani H, Dynamic network traffic assignment and simulation methodology for advanced system management applications. Networks and Spatial Economics, 1, 3-4, pp. 267-292, 2001.

[5] Polimeni, A. and Vitetta, A., The role of ITS in evacuation route optimization for emergency vehicles. WIT Transactions on Information and Communication Technologies, 44, pp. 517-529, 2012.

[6] De Maio, M.L., Musolino, G. and Vitetta, A., The role of ITS in evacuation route choice. WIT Transactions on Information and Communication Technologies, 44, pp. 503-515, 2012.

[7] Chen, A., Pravinvongvuth, S. and Chootinan, P., Scenario-based multiobjective AVI reader location models under different travel demand patterns. Transportmetrica, 6(1), pp. 53-78, 2010.

[8] Torday, A. and Dumont, A.G. Probe vehicle based travel time estimation in urban networks. In: TRISTAN V, 2004.

[9] Maerivoet, S., Daems, F., Maertens, F., Renckens, K., Van Houtte, P. and Buelens, L., A field trial on smart mobility. Procedia - Social and Behavioral Sciences, 54, pp. 926 - 935, 2012.

[10] Dong C. and Paty C. S., Application of adaptive weights to intelligent information systems: an intelligent transportation system as a case study. Information Sciences, 181, pp. 5042-5052, 2011.

[11] Ando N. and Taniguchi E., Travel time reliability in vehicle routing and scheduling with time windows. Netw Spat Econ, 6, pp. 293-311, 2006.

[12] Iera A., Modafferi A., Musolino G. and Vitetta A. An experimental station for real-time traffic monitoring on an urban road. The IEEE $5^{\text {th }}$ International Conference on Intelligent Transportation Systems, 2002.

[13] Vitetta, A., Musolino, G. and Marcianò, F.A., Safety of users in road evacuation: Supply and demand-supply interaction models for users. WIT Transactions on the Built Environment, 96, pp. 783-792, 2007.

[14] Vitetta, A., Musolino, G. and Marcianò, F.A., Safety of users in road evacuation: Calibration of cost functions and simulation. WIT Transactions on the Built Environment, 101, pp. 715-725, 2008.

[15] Polimeni, A. and Vitetta, A., Optimising waiting at nodes in timedependent networks: cost functions and applications. Journal of Optimization Theory and Applications, 156(3), pp. 805-818, 2013.

[16] Musolino, G. and Vitetta, A., Short-term forecasting in road evacuation: calibration of a travel time function. WIT Transactions on The Built Environment, 116, pp. 615-626, 2011. 
[17] Russo, F. and Vitetta, A., Reverse assignment: calibrating link cost functions and updating demand from traffic counts and time measurements. Inverse Problems in Science and Engineering, 19 (7), pp. 921-950, 2011.

[18] Emmerink R.H.M., Axhausen K.W., Ijkamp P. and Rietveld P., The potential of information provision in a simulated road transport network with non-recurrent congestion. Transp. Res. C, 3(5), pp. 293-309, 1995.

[19] Beynon-Davies P., Human error and information systems failure: the case of the London ambulance service computer-aided despatch system project. Interacting with Computers, 11, pp. 699-720, 1999.

[20] Derekenaris G., Garofalakis J., Makris C., Prentzas J., Sioutas S. and Tsakalidis A., Integrating GIS, GPS and GSM technologies for the effective management of ambulances. Comput., Environ. and Urban Systems, 25, pp. 267-278, 2001.

[21] Dixon C.S. and Haas R., GNSS user requirement in emergency management. Proc. of RIN NAV '08 Conference, 2008.

[22] Russo, F. and Chilà G., Safety of users in road evacuation: Modelling and DSS for demand. WIT Transactions on Ecology and the Environment, 120, pp. 465-474, 2009.

[23] Russo, F. and Chilà, G., Safety of users in road evacuation: demand models. WIT Transactions on the Built Environment, 96, pp. 773-782, 2007.

[24] Russo, F. and Chilà G., Safety of users in road evacuation: RP vs. SP surveys in demand analysis. WIT Transactions on the Built Environment, 101, pp. 703-713, 2008.

[25] De Maio, M. L., Musolino, G. and Vitetta, A., Traffic assignment models in road evacuation. The Sustainable City VII, pp.1041-1051, 2012.

[26] Polimeni, A. and Vitetta, A., Joint network and route optimization in road evacuation. WIT Transactions on Ecology and the Environment, 155, pp. 1053-1065, 2011. 